\title{
Are we alone? Stories from the frontline of Kepler's search for Earth's twin (Presentation Video)
}

Jon Jenkins

Jon Jenkins, "Are we alone? Stories from the frontline of Kepler's search for Earth's twin (Presentation Video)," Proc. SPIE 8864, Techniques and Instrumentation for Detection of Exoplanets VI, 886404 (17 October 2013); doi: $10.1117 / 12.2031741$

EDIE Event: SPIE Optical Engineering + Applications, 2013, San Diego, California, United States 


\title{
Are We Alone? Stories from the Frontline of Kepler's Search for Earth's Twin (Presentation Video)
}

\author{
Jon Jenkins, SETI Institute/NASA Ames Research Ctr. (United States)
}

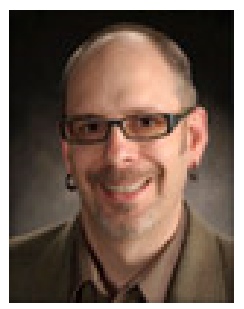

\begin{abstract}
Kepler vaulted into the heavens on March 7, 2009, initiating NASA's search for Earth-size planets orbiting Sun-like stars in the habitable zone, where liquid water could exist on the planetary surface and support alien biology. Never before has there been a photometer capable of reaching a precision near $20 \mathrm{ppm}$ in 6.5 hours while conducting nearly continuous and uninterrupted observations for several years. The flood of exquisite photometric data over the last 4 years on $190,000+$ stars has provoked a watershed of results. Over 2,700+ candidate planets have been identified of which an astounding 1171 orbit 467 stars. Over 120+ planets have confirmed or validated and the data have also led to a resounding revolution in asteroseismology. Recent discoveries include Kepler-62 with 5 planets total of which 2 are in the habitable zone, and are 1.4 and 1.7 times the radius of the Earth. Designing and building the Kepler photometer and the software systems that process and analyze the resulting data presented a daunting set of challenges, including how to manage the large data volume, how to detect miniscule transit signatures against stellar variability and instrumental effects, and how to review hundreds of diagnostics produced for each of $\sim 20,000$ candidate transit signatures. The challenges continue into flight operations, as the photometer and spacecraft have experienced aging and changes in hardware performance over the course of time. The success of Kepler sets the stage for TESS, NASA's next mission to detect Earth's closest cousins.
\end{abstract}

View presentation video on SPIE's Digital Library: http://dx.doi.org/10.1117/12.2031741 